\title{
Comparision of Live Videotapes/Charts V/S Verbal Instruction in Osteoarthritis Knee.
}

\author{
Dr.Manoj Agnihotri ${ }^{1}$, Dr. Medha Deo ${ }^{2}$, Dr.Sana Siddique ${ }^{3}$ \\ ${ }^{1}$ Associate Professor, \\ ${ }^{2}$ Principal Cum Professor \\ ${ }^{3}$ Ex BPTh Student \\ Terna Physiotherapy College,Nerul West Navi Mumbai
}

\section{Introduction}

Osteoarthritis (OA) is a degenerative joint disease; also commonly known as wear and tear arthritis, is a condition in which the natural cushioning between joints cartilage wear away. When this happens, the bones of the joints rub more closely against one another with less of the shock-absorbing benefits of cartilage. The rubbing results in pain, swelling, stiffness, decreased ability to move and, sometimes, the formation of bone spurs.

Osteoarthritis of knee is the most common type of arthritis. While it can occur even in young people, the chance of developing osteoarthritis rises after age 45. According to the Arthritis Foundation, most of the populations have osteoarthritis, with the knee being one of the common affected areas. Women are more likely to have osteoarthritis than men.

The causes of knee osteoarthritis:-Several factors increase the risk of developing significant arthritis at an earlier age: Age, Weight, Heredity, Gender, and Repetitive stress injuries.

The videotapes/charts for home program for patients with OA Knee is used effectively for getting better results because the videotapes/charts gives the necessary instructions which is through visual as well as auditory pathway which resulted to be part of proper rehabilitation program which is followed properly and gives good results. Even the errors during the exercise regime decreases.

On the other hand verbal commands are also there. But over there chances of performing exercises in their own pattern which they have understood, misconception and the most important thing is how well is the understanding and learning exercises regime of patients which varies from person to person and recalling them is also difficult which is usually see in the old age group. At times as the age advances communication becomes barriers because of hearing issues and behavioral problems which results into performing exercises that exercises which they wants according to their wish and the things which they can recall.

\section{Review Of Literature}

- Nguyen C, Lefevre-Colau MM, Poiraudeau S, Rannou F et al did study on Rehabilitation (exercises and strength training) and osteoarthritis and he concluded that OA widely includes land and water based exercise, strength training, weight management, self-management and education, biomechanical interventions and physically active lifestyle. Exercise therapy and specific strengthening exercise or strengthen training for the lower limbs reduce pain and improve physical function in OA knee.

- Alnahdi AH,Zeni Ja,Snyder-Mackler et al did study on muscle which should be targeted in patients with OA knee and concluded that the relationship between quadriceps and other muscle around the knee should be strengthened and progression and determine the optimal exercise prescription.

- Coudeyre E,Jegu AG,Giustanini M,Marrel JP,Edouard P,PereireB et al did study on isokinetic muscle strengthening for OA knee and concluded that isokinetic muscle training is an effective way to propose dynamic muscle strengthening for OA knee rehabilitation and has significant effect on pain and disability.

- Carolyn J.PAGE, Rana S.HINMAN and Kim L.BENNELL et al did study on physiotherapy management of knee OA and concluded that there is a strong evidence treatment outcome including group or home exercises are all effective, although therapist contact may improve benefits for OA knee.

- Marlene Franosen,Sara McConnell et al did study in exercise for OA knee and concluded that land based therapeutic exercises has at least short term benefit in term of reduced knee pain and improved physical functions for OA knee. The magnitude of the treatment effect would be considered small, but comparable to estimates for non-steroidal anti-inflammatory drugs. 


\section{Aim And Objectives}

Aim

To know the effect of live videotapes/charts v/s verbal instructions of knee exercises for treating patients of osteoarthritis of knee.

\section{Objectives}

$>$ To know pre and post effect of live videotapes/charts for OA knee patients.

$>$ To know the pre and post effect of verbal instruction.

$>$ To compare post effects of videotapes v/s verbal instructions.

Type of study:-Survey based.

\section{Methodology}

Study set up: -Terna Hospital Research Centre, Nerul.

Navi Mumbai Municipal Co-orporation, Vashi.

Home Based

Study Design:-Randomized.

Sample size:-30 patients with 4-5 pain On VAS scale.

These patients were randomly assigned into one of the two treatment group i.e. 15each.

Inclusion Criteria:-

OA knee (TF/PF) in the age group of 50-60 years.

Exclusion Criteria:-

$>$ OA knee patients under age group i.e. less than 50 .

$>$ Neurologically disorder patients.

$>$ Recent \# of lower limb.

$>$ Recent cardiac surgery.

Outcome measures:-KOOS Knee Survey.

Procedure:- The patients were divided into 2 groups and below mentions exercises were given.

Group A

(15 patients)

Videotapes/charts of knee exercises

for OA Knee (TF/PF)( no instructions)

Knee exercises

*Static Quadriceps.

*Static Hamstrings.

* Dynamic knee extension.

*Straight leg raising.

*Weight bearing closed kinematics exs.

(squatting, steps up, lunges)
Group B

(15 patients)

Verbal instructions of knee exercises

Knee exercises

*Static Quadriceps.

*Static Hamstrings

*Dynamic knee extension.

*Straight leg raising.

*Weight bearing closed kinematic exs. (squatting, stepsup,lunges)

Frequency:-2 weeks regime was given to the patients of both the group. After that patients were either called at OPD for re evaluation or else therapist had gone to patients home for data collection.

\section{Statistical Analysis}

1. Paired t test:-This was used to compare pre \& post VAS and KOOS of OA patients with videotapes/charts.

2. Unpaired $t$ test:-This was used to compare post score of VAS and KOOS of different group.

3. The mean and SD values were noted and $\mathrm{p}$ values were recorded.

GRAPH 1:-Comparison of mean VAS pre and post treatment in group A (Videotapes) using paired t test.

Pre and post VAS in Group A

Mean and Standard Deviation.

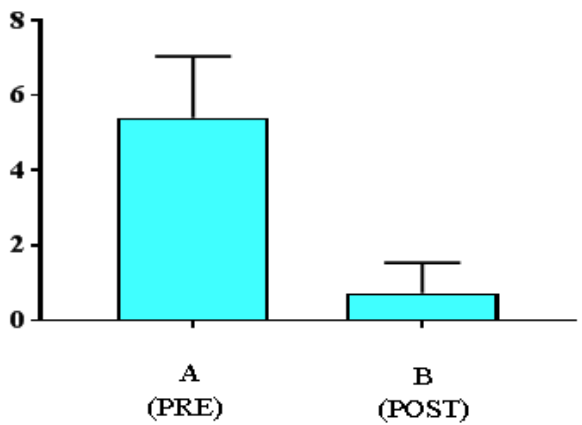




\begin{tabular}{|l|l|}
\hline & GROUP A \\
\hline Pre Treatment & 4.667 \\
\hline Post Treatment & 1.175 \\
\hline
\end{tabular}

\section{P VALUE}

$P$ value $<0.001$

(extremely significant)

\section{Inference:}

The VAS had reduced significantly after giving videotapes/charts for the treatment.

GRAPH 2:-Comparison of mean VAS pre and post treatment in Group B (Verbal commands) using paired $t$ test.

Pre and post VAS in Group B

Mean and $S$ tandard Deviation.

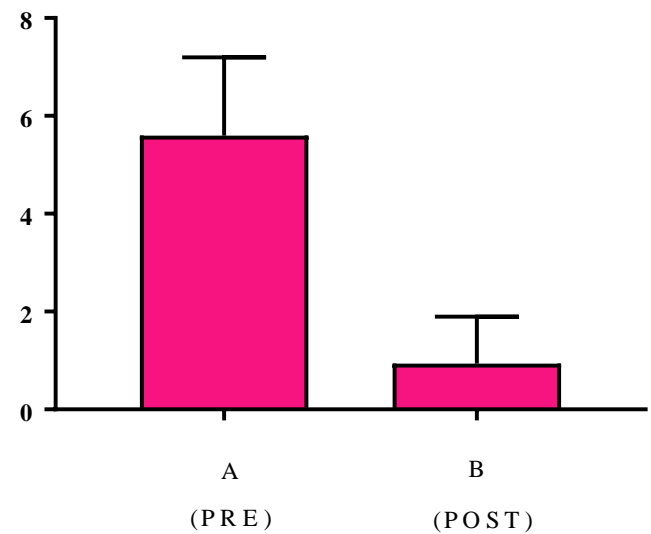

\begin{tabular}{|l|l|}
\hline & GROUP B \\
\hline Pre Treatment & 4.667 \\
\hline Post Treatment & 1.345 \\
\hline
\end{tabular}

\begin{tabular}{|l|}
\hline P VALUE \\
\hline P value $<0.001$ \\
(extremely significant) \\
\hline
\end{tabular}

\section{Inference:}

The VAS as reduced significantly after giving verbal instructions for the treatment.

GRAPH 3:-Comparison of mean VAS post treatment between group A and group B using unpaired t test.

Post treatment VAS of Group A and B

Mean and Standard Deviation.

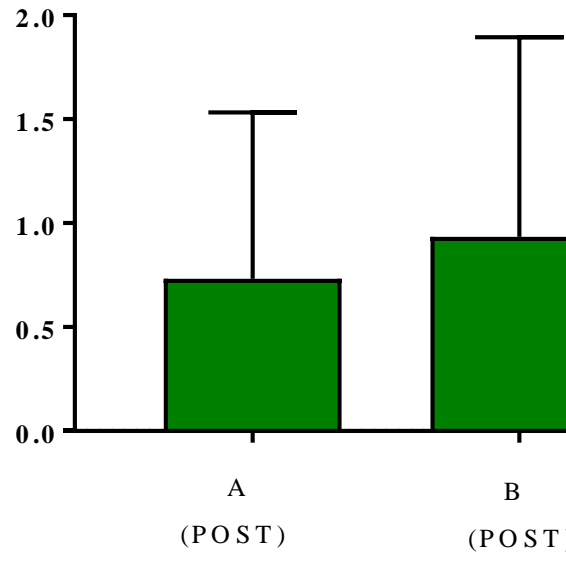


Comparision of Live Videotapes/Charts V/S Verbal Instruction in Osteoarthritis Knee.

\begin{tabular}{|l|l|l|}
\hline & Post Treatment & P value \\
\hline Group A & 0.779 & $\begin{array}{l}\text { P value }<0.001 \\
\text { (extremely significant) }\end{array}$ \\
\hline Group B & 0.830 & \\
\hline
\end{tabular}

Inference:

VAS scores were significantly reduced in both the groups.

GRAPH 4:-Comparision of mean KOOS score pre and post treatment in Group A(Videotapes) using paired $\mathrm{t}$ test.

Pre and post KOOS in Group A

Mean and Standard Deviation.

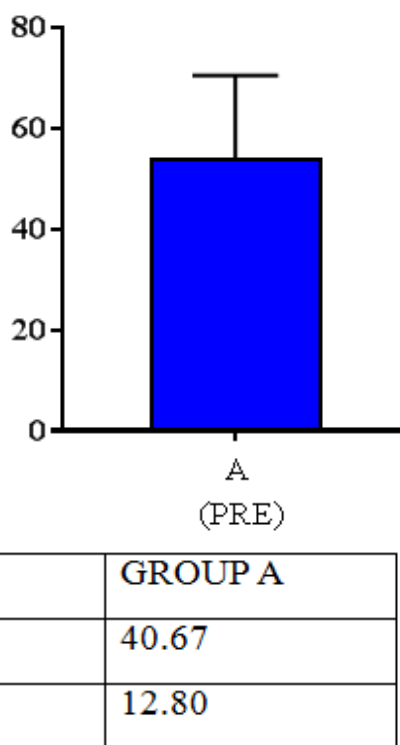

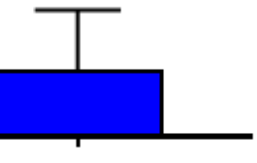

$\mathrm{B}$

(POST)

\begin{tabular}{|l|}
\hline P VALUE \\
\hline $\begin{array}{l}\text { P value }<0.001 \\
\text { (extremely significant) }\end{array}$ \\
\hline
\end{tabular}

Inference:-

The KOOS score had significantly reduced post treatment shown by videotapes.

GRAPH 5:-Comparision of mean KOOS score pre and post treatment in Group B (Verbal instructions) using paired t test.

Pre and post KOOS score in Group B

Mean and Standard Deviation.

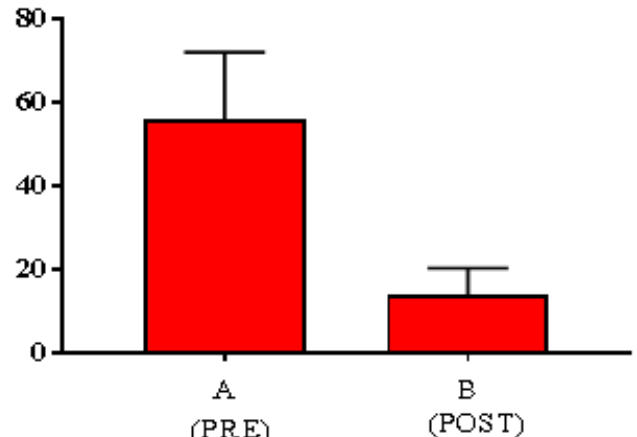

\begin{tabular}{|c|c|}
\hline & GROUP B \\
\hline Pre treatment & 42 \\
\hline Post treatment & 12.65 \\
\hline
\end{tabular}

\section{P VALUE}

P value $<0.001$

(extremely significant) 
Inference:-

The KOOS score had reduced significantly post treatment after verbal instruction.

GRAPH 6:-Comparision of mean KOOS score post treatment Group A and B using unpaired t test.

Post treatment KOOS score of Group A and B

Mean and Standard Deviation.

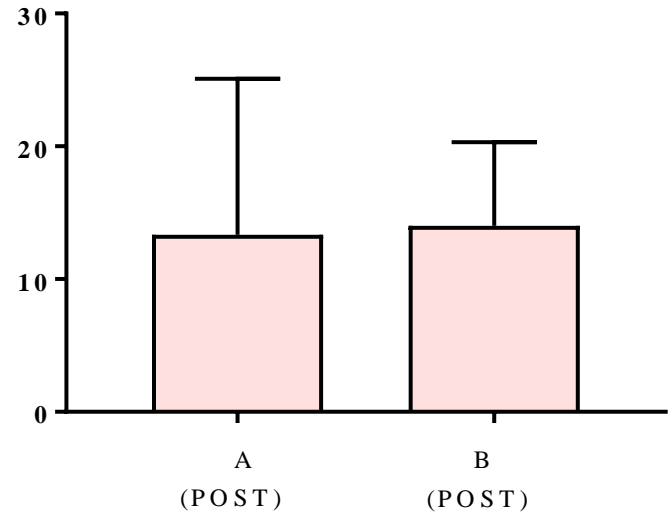

\begin{tabular}{|l|l|l|}
\hline & Post Treatment & P value \\
\hline Group A & 27.713 & $\begin{array}{l}\text { P value }<0.001 \\
\text { (extremely significant) }\end{array}$ \\
\hline Group B & 29.30 & \\
\hline
\end{tabular}

Inference:-

KOOS score was significantly reduced in the videotapes/charts group.

\section{Discussion}

The purpose of the study was to compare the effect of videotapes/charts versus verbal commands in osteoarthritis patients. There was a statistically significant decline of pain in both the groups which proves that the efficacy of both videotapes/charts and verbal commands decreased the VAS and KOOS score in patients with osteoarthritis knee.

The exercise given to both the groups was same and even the duration of treatment regime was same i.e. 2 weeks. There was significant improvement seen in VAS and KOOS score. Research suggests that people learn abstract, new and novel concepts more easily when they are presented both verbal and visual form (Salomon, 1979).Other empirical research also shows it more accessible to persons and later recall it because visual media helps in retaining concepts and ideas. Videotapes are very high capable showcasing complex ideas in short span of time which offers both cognitive and affective experiences. Videotapes/charts takes (15-20 mins or less then) helps to focus attention while watching the media and develops analytical skills in applying what they are learning which makes it easier them to perform. Videotapes/charts gave them proper instructions of the exercise which should be followed through visual as well as auditory feedback and made their task easier.

On the other hand verbal learning is associated with the memorization and retention of lists of words, in order to describe basic elements of associative learning. The type of mental events that occur in verbal learning studies go beyond passive memorization, as learners can play a very active role in manipulating experimental stimuli. Since the movements carried on during the exercise regime resembled the movements which occur during most of the activities of daily living, this might be the probable reason for the improvement in functional capability as seen with KOOS score. The reduction of KOOS score indicated that there was improvement of function in both the groups after 2 weeks. Hence the study and statistical analysis suggested that videotapes/charts gave better results as compared to verbal commands in the same treatment duration.

\section{Conclusion}

Videotapes/charts and verbal instruction both seems to be effective in treating OA patients. But it is noticed that videotapes/charts is more better than verbal instructions. 


\section{Bibliography}

[1]. Rehabilitation (exercise and strength training) and osteoarthritis Journal (2014):-Nguyen C, Lefevre-Colau MM, Poiraudeau S, Rannou F.

[2]. Knee osteoarthritis Journal (2011):-Alnahdi AH, Zeni Ja, Snyder-Mackler.

[3]. Isokinetic muscle strengthening for OA knee; International Journal:-Coudeyre E,Jegu AG,Giustanini M,Marrel JP,Edouard P,PereireBisokinetic.

[4]. International Journal of Osteoarthritis Diseases(2011):-Carolyn J.PAGE,Rana S.HINMAN and Kim L.BENNEL

[5]. Marlene Franosen,Sara McConnell (2015)

\section{Limitations}

[1]. Small sample size.

[2]. There was treatment regime of only 2 weeks.

[3]. The videotapes audio was muted for the people how could not understand English. 